\title{
The C. elegans 3' UTRome v2 resource for studying mRNA cleavage and polyadenylation, 3 '-UTR biology, and miRNA targeting
}

\author{
Hannah S. Steber, ${ }^{1,2}$ Christina Gallante, ${ }^{3}$ Shannon O'Brien, ${ }^{2}$ Po-Lin Chiu, ${ }^{4}$ \\ and Marco Mangone 1,2,5 $^{1}$ \\ ${ }^{1}$ Molecular and Cellular Biology Graduate Program, School of Life Sciences, Tempe, Arizona 85287; ${ }^{2}$ Virginia G. Piper Center for \\ Personalized Diagnostics, The Biodesign Institute at Arizona State University, Tempe, Arizona 85281, USA; ${ }^{3}$ Barrett, The Honors \\ College, Arizona State University, Tempe, Arizona 85281, USA; ${ }^{4}$ Center for Applied Structural Discovery, The Biodesign Institute at \\ Arizona State University, Tempe, Arizona 85287, USA
}

\begin{abstract}
3' Untranslated regions ( $3^{\prime}$ UTRs) of mRNAs emerged as central regulators of cellular function because they contain important but poorly characterized cis-regulatory elements targeted by a multitude of regulatory factors. The model nematode Caenorhabditis elegans is ideal to study these interactions because it possesses a well-defined 3' UTRome. To improve its annotation, we have used a genome-wide bioinformatics approach to download raw transcriptome data for 1088 transcriptome data sets corresponding to the entire collection of C. elegans trancriptomes from 2015 to 2018 from the Sequence Read Archive at the NCBI. We then extracted and mapped high-quality $3^{\prime}$-UTR data at ultradeep coverage. Here, we describe and release to the community the updated version of the worm $3^{\prime}$ UTRome, which we named $3^{\prime}$ UTRome v2. This resource contains high-quality $3^{\prime}$-UTR data mapped at single-base ultraresolution for 23,084 3'-UTR isoform variants corresponding to 14,788 protein-coding genes and is updated to the latest release of WormBase. We used this data set to study and probe principles of mRNA cleavage and polyadenylation in C. elegans. The worm 3' UTRome v2 represents the most comprehensive and high-resolution $3^{\prime}$-UTR data set available in $C$. elegans and provides a novel resource to investigate the mRNA cleavage and polyadenylation reaction, $3^{\prime}$-UTR biology, and miRNA targeting in a living organism.
\end{abstract}

[Supplemental material is available for this article.]

3' Untranslated regions (3' UTRs) are the portions of mRNA located between the end of the coding sequence and the poly(A) tail of RNA polymerase II-transcribed genes. They contain cis-regulatory elements targeted by miRNAs and RNA-binding proteins and modulate mRNA stability, localization, and overall translational efficiency (Bartel 2018). Because multiple 3'-UTR isoforms of a particular mRNA can exist, differential regulation of $3^{\prime}$ UTRs has been implicated in numerous diseases, and its discriminative processing influences development and metabolism (Mayr and Bartel 2009; Zhu et al. 2018). 3' UTRs are processed to full maturity through cleavage of the nascent mRNA and subsequent poly(A) tail addition to its $3^{\prime}$ end by the nuclear poly(A) polymerase enzyme (PABPN1) (Kühn and Wahle 2004). The mRNA cleavage step is a dynamic regulatory process directly involved in the control of gene expression in eukaryotes. The reaction depends on the presence of a series of sequence elements located within the end of the $3^{\prime}$ UTRs. The most well-characterized sequence is the poly(A) signal (PAS) element, a hexameric motif located at $\sim 19$ nt from the polyadenylation site in the $3^{\prime}$ UTR of mature mRNAs. In metazoans, the PAS element is commonly "AAUAAA," which accounts for more than half of all 3'-end processing in eukaryotes (Mangone et al. 2010; Tian and Graber 2012), although alternative forms of the PAS elements exist (Mangone et al. 2010; Jan et al. 2011; Blazie et al.

\footnotetext{
${ }^{5}$ Present address: Biodesign Institute, Arizona State University, Tempe, AZ 85281, USA

Corresponding author: mangone@asu.edu

Article published online before print. Article, supplemental material, and publication date are at http://www.genome.org/cgi/doi/10.1101/gr.254839.119. Freely available online through the Genome Research Open Access option.
}

2015). Previous studies have shown that single-base substitutions in this sequence reduce the effectiveness of the cleavage and the polyadenylation of the mRNA transcript (Sheets et al. 1990; Chen et al. 1995). However, this canonical sequence is necessary and sufficient for efficient 3 '-end polyadenylation in vitro (Clerici et al. 2018; Sun et al. 2018). A less defined "GU-rich" element is also known to be present downstream from the cleavage site to facilitate the cleavage and polyadenylation steps (Chen et al. 1995). Recently, studies in human cells identified an additional upstream "UGUA" sequence that is not always present and not required for the cleavage process, but can act as a cleavage enhancer in the context of alternative polyadenylation (APA) if present (Zhu et al. 2018).

APA is a poorly understood mRNA maturation step that produces mRNAs with different 3 '-UTR lengths owing to the presence of multiple PAS elements within the same $3^{\prime}$ UTR. The usage of the most upstream element, termed the proximal PAS element, leads to the formation of shorter 3'-UTR isoforms, whereas the use of the distal PAS element results in a longer isoform. These changes in size may include or exclude regions to which regulatory molecules such as microRNAs (miRNAs) and RNA-binding proteins (RBPs) can bind, substantially impacting gene expression (Matlin et al. 2005; Bartel 2009). Although its function in eukaryotes is still not fully understood, a recent study revealed that APA may occur in a tissue-specific manner and, at least in the nematode Caenorhabditis elegans, is used in specific cellular contexts to evade

(C) 2019 Steber et al. This article, published in Genome Research, is available under a Creative Commons License (Attribution-NonCommercial 4.0 International), as described at http://creativecommons.org/licenses/by-nc/4.0/. 
miRNA-based regulatory networks in a tissue-specific manner (Blazie et al. 2015, 2017).

The length of 3' UTRs is defined during the cleavage and polyadenylation reaction, which is still poorly characterized in metazoans. Although it involves a multitude of proteins and is considered to be very dynamic, the role of each member of the complex and the order in which this process is executed is still not fully understood.

In humans, the cleavage and polyadenylation complex (CPC) is composed of at least 17 proteins (Fig. 1A) that immunoprecipitate into at least four large subcomplexes: the cleavage and polyadenylation specificity factor (CPSF), the cleavage stimulation factor (CstF), the cleavage factor Im (CFIm), and the cleavage factor IIm (CFIIm) subcomplexes (Fig. 1A). The CPSF subcomplex forms the minimal core component necessary and sufficient to recognize and bind the PAS element of the nascent mRNA in vitro (Fig. 1A; Tian and Manley 2017). In humans, the CPSF subcomplex is composed of the proteins CPSF1 (also known as CPSF160) (Clerici et al. 2017; Sun et al. 2018), CPSF2 (also known as CPSF100) (Mandel et al. 2006), CPSF3 (also known as CPSF73) (Mandel et al. 2006), CPSF4 (also known as CPSF30) (Clerici et al. 2017; Sun et al. 2018), FIP1L1 (Kaufmann et al. 2004), and WDR33 (Clerici et al. 2017; Sun et al. 2018). Initial experiments assigned CPSF1 with the role of binding the PAS element, but it is now clear that WDR33 and CPSF4 are the proteins that instead contact the PAS directly. CPSF1 has a scaffolding role in this process and keeps this subcomplex structured (Chan et al. 2014). The interaction between members of the CPSF core complex (WDR33, CPSF4, and CPSF1) and the PAS element was recently revealed using singleparticle cryo-EM (Clerici et al. 2017; Sun et al. 2018), showing a unique conformation in which the PAS element twists to form an S-shaped structure with a noncanonical pairing between the U3 and the A6 in the PAS element (Sun et al. 2018).

CPSF3 is the endonuclease that performs the cleavage of the nascent mRNAs (Fig. 1A; Ryan et al. 2004; Mandel et al. 2006). CPSF3 is also required in the cleavage of pre-histone mRNAs and is recruited on their cleavage site by the RNU7-1 snRNP (Yang et al. 2009).

The CstF subcomplex is the second most well-characterized subcomplex involved in the cleavage and polyadenylation reaction (Fig. 1A). CstF binds to GU-rich elements located downstream from the cleavage site in the nascent mRNA and directly contacts the CPSF subcomplex using its conserved HAT-C domain (Fig. 1A; Bai et al. 2007; Yang et al. 2018). The CstF subcomplex is a trimer of heterodimers composed of CSTF3 (also known as CstF-77), CSTF2 (also known as CstF-64), and CSTF1 (also known as CstF-50) (Yang et al. 2018). CSTF3 holds the complex together through its Pro-rich domain located on its C-terminal region (Fig. 1A; Takagaki and Manley 2000). CSTF2 recognizes GU-rich sequences through its N-terminal RRM domain (Pérez Cañadillas and Varani 2003; Yang et al. 2018) and interacts with CSTF3 and the scaffolding protein symplekin using its N-terminal HINGE domain (Fig. 1A; Takagaki and Manley 2000).

The CFIm and CFIIm subcomplexes are less characterized (Fig. 1A). The CFIm subcomplex is composed of the CPSF6 (also known as CFIm68), CPSF7 (also known as CFIm59), and NUDT21 (also known as CFIm25) subunits, and it was recently shown to contribute to APA by influencing PAS selection (Martin et al. 2012; Hwang et al. 2016). NUDT21 binds the "UGUA" RNA element upstream of the cleavage site and contributes to 3 '-end processing and APA by recruiting CPSF7 and CPSF6 (Yang et al. 2010, 2011; Zhu et al. 2018).
Despite the importance of this complex, the CPC remains poorly characterized in most species, including humans, and most of the research in this field is performed in vitro.

The roundworm nematode C. elegans represents an attractive, novel system to study the cleavage and polyadenylation process in vivo. Most of the CPC is conserved between humans and nematodes, including known functional domains and protein interactions (Fig. 1B; Supplemental Fig. S1). C. elegans possess the most well-annotated $3^{\prime}$ UTRome available so far in metazoans, with $\sim 26,000$ mapped $3^{\prime}$-UTR boundaries corresponding to $\sim 16,000$ distinct C. elegans protein-coding genes (Mangone et al. 2010; Jan et al. 2011).

The C. elegans 3' UTRome was originally developed in 2011 within the modENCODE Project (Mangone et al. 2008, 2010; Gerstein et al. 2010) and represented a milestone in 3'-UTR biology because it allowed the community to study and identify important regulatory elements such as miRNA and RBP targets with great precision. A second 3' UTRome was later published using a different mapping pipeline (Jan et al. 2011), confirming most of the previous data such as isoform numbers and PAS usage, and so forth. Other data sets were made available later, mostly focusing on tissue-specific $3^{\prime}$ UTRs and alternative polyadenylation (Haenni et al. 2012; Blazie et al. 2015, 2017; Chen et al. 2017; Diag et al. 2018; West et al. 2018).

Although refined and based on several available data sets, only a subset of $C$. elegans $3^{\prime}$ UTRs in protein-coding genes are sufficiently annotated today, and the existing mapping tools do not yet reach the single-base resolution necessary to execute downstream analysis and study the cleavage and polyadenylation process in detail. Most of these $3^{\prime}$-UTR data sets were developed using a gene prediction set now considered obsolete (WS190), and the $3^{\prime}$-UTR coordinates often do not match the new gene coordinates.

To address these and other issues, we developed a novel pipeline to bioinformatically extract 3'-UTR data from almost all C. elegans transcriptome data sets stored in the public repository Sequence Read Archive (SRA) trace archive. This blind approach produced a new saturated data set we named $3^{\prime}$ UTRome v2, which is available to the community as additional JBrowse and gBrowse tracks in the C. elegans database WormBase (https:// www.WormBase.org) (Stein et al. 2001; Lee et al. 2018) and in the 3'-UTR-centric database 3' UTRome (http://www.UTRome .org) (Mangone et al. 2008, 2010). We also used this data set to study the PAS sequence requirement and the cleavage location of the CPC in vivo using transgenic C. elegans animals.

\section{Results}

Functional elements of the human cleavage and polyadenylation complex are conserved in nematodes

To initially gain structural and functional information for the C. elegans CPC, we downloaded the protein sequences of the orthologs of the C. elegans CPC and aligned them to their human counterparts (Fig. 1B; Supplemental Fig. S1). Based on sequence similarity, C. elegans possess orthologs to all the known members of the human CPC, with many peaks of conservation interspersed within known interaction domains of the subunits. The amino acids that make direct contact with PAS elements are also conserved in C. elegans; 11 of the 12 amino acids that form hydrogen bonds and salt bridges with the PAS element (Clerici et al. 2017) are present in both the CPSF4 and WDR33 worm orthologs CPSF-4 and PFS-2 (V67 ${ }^{\text {CPSF } 4}$ with V81 ${ }^{\text {CPSF-4 }} ; \quad$ K69 6 CPSF4 with $\mathrm{K} 83^{\text {CPSF-4 }}$; 
A

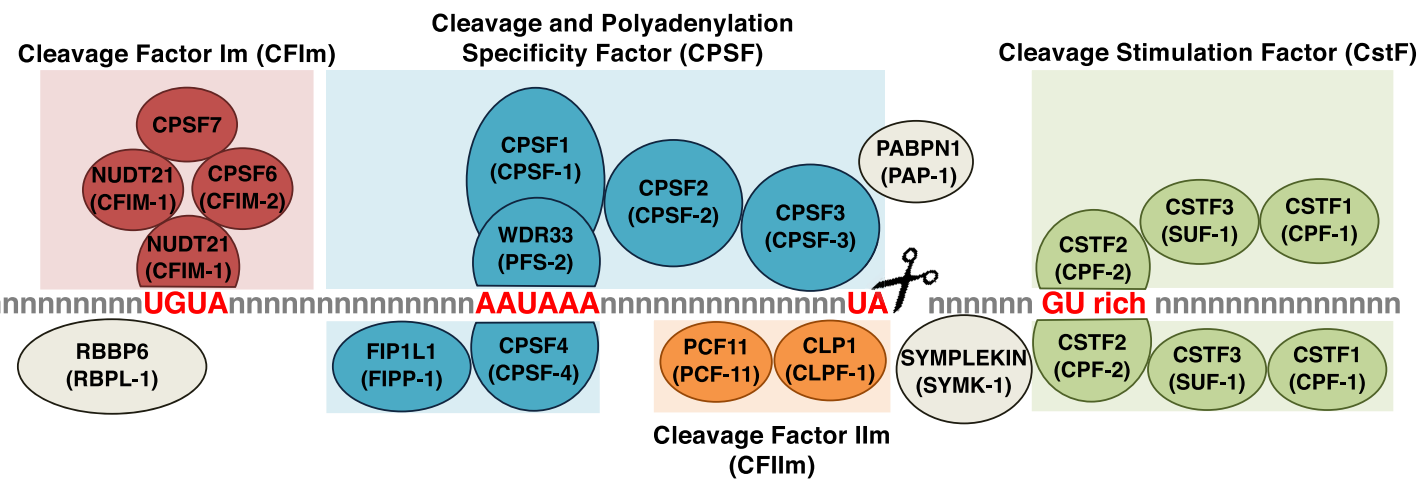

B

CPSF1 (CPSF-1)

WDR33 (PFS-2)
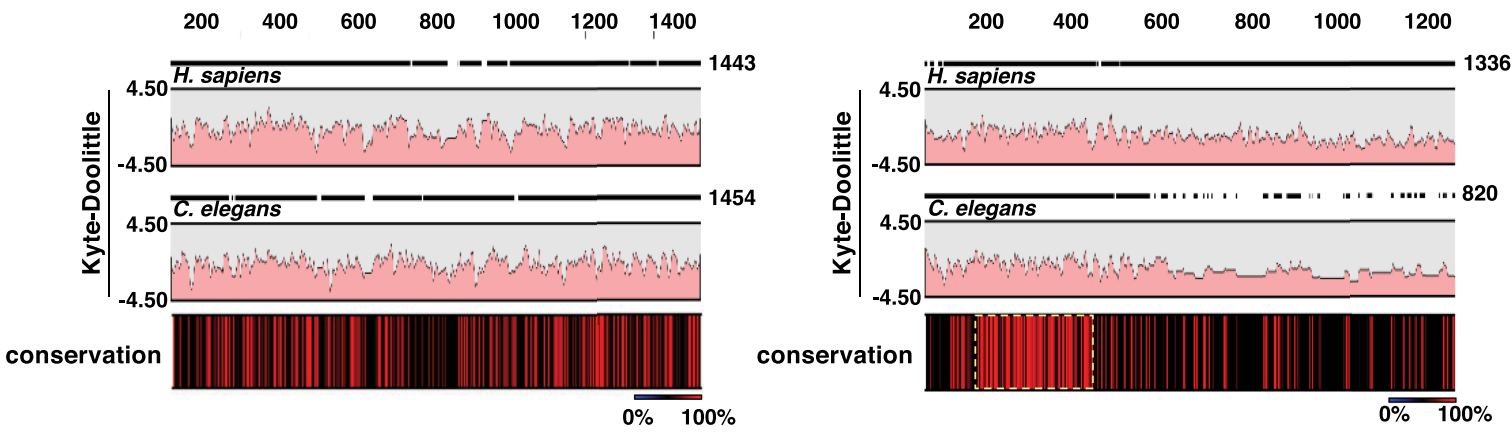

conservation

CPSF3 (CPSF-3)

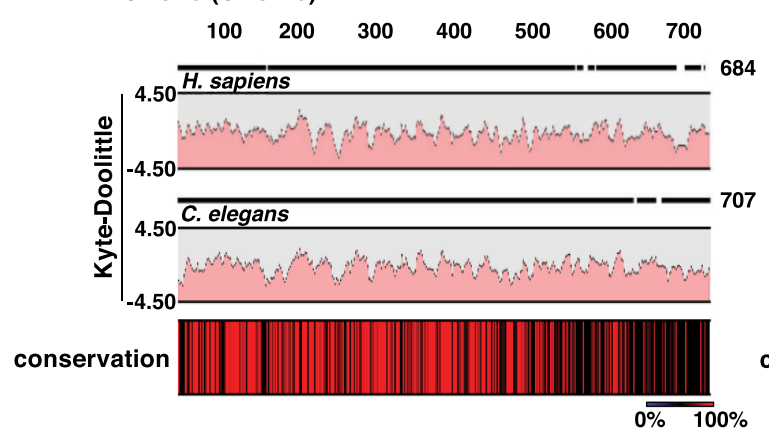

C

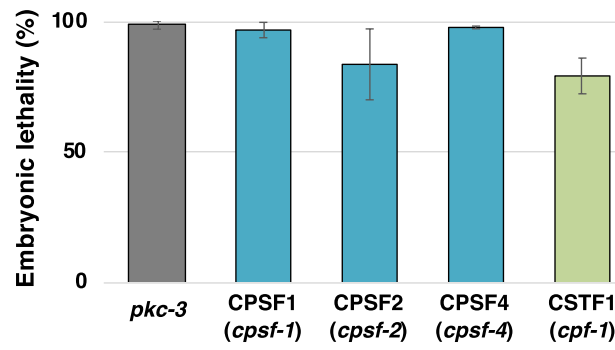

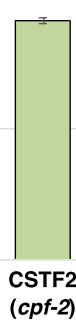

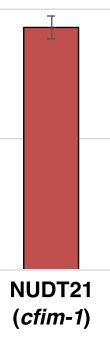

(cfim-2)

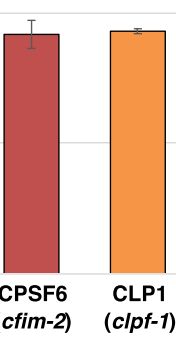

CPSF4 (CPSF-4)
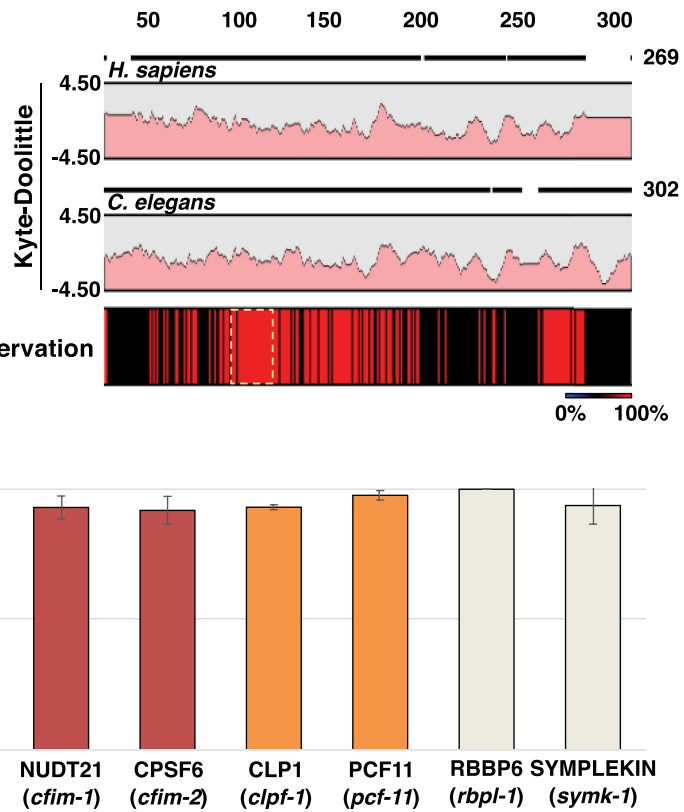

Figure 1. The C. elegans members of the cleavage and polyadenylation complex (CPC). (A) The CPC is composed of at least four independent subcomplexes named cleavage and polyadenylation specificity complex (blue), which canonically recognizes the PAS hexamer "AAUAAA" and performs the cleavage downstream from the dinucleotide TA; the cleavage stimulation factor complex (green), which binds downstream from the cleavage site to GU-rich elements; and the cleavage factor CFIm (red) and CFIIm (orange) complexes. CFIm recognizes the element "UGUA" located upstream of the PAS element. This element is not always present. Other known required factors are the poly $(A)$ polymerase enzyme, the scaffolding member symplekin, and RBBP6. The names of the C. elegans orthologs are shown in parentheses. (B) The human and C. elegans CPSF subcomplexes are similar in amino acid composition and structure. Two-species alignments between several members of the human and C. elegans CPSF members. Amino acids $100 \%$ conserved between these two species are shown in red in the conservation bar. Yellow dashed boxes show the sequence of the proteins that interact with the PAS element. Functional domains are conserved. The two Kyte-Doolittle graphs in each panel indicate the hydrophobic amino acids in human and C. elegans. (C) RNAi was used to selectively silence most of the members of the CPC complex in C. elegans. We observed a strong embryonic lethality phenotype with all the RNAi experiments performed.

\section{Genome Research}

www.genome.org 
R73 ${ }^{\text {CPSF } 4}$ with R87 ${ }^{\text {CPSF-4 }}$; E95 ${ }^{\text {CPSF4 }}$ with E109 ${ }^{\text {CPSF-4 }}$; K77 ${ }^{\text {CPSF4 }}$ with K91 ${ }^{\mathrm{CPSF}-4}$; S106 ${ }^{\mathrm{CPSF} 4}$ with S120 ${ }^{\mathrm{CPSF}-4}$; N107 ${ }^{\mathrm{CPSF} 4}$ with N121 ${ }^{\mathrm{CPSF}-4}$; R54 $4^{\text {WDR33 }}$ with R80 ${ }^{\text {PFS-2 }}$; R47 $7^{\text {WDR33 }}$ with R71 ${ }^{\text {PFS-2 }}$; R49 $9^{\text {WDR33 }}$ with R73 ${ }^{\text {PFS-2 }}$ ) (Fig. 1B; Supplemental Fig. S1). The only exception is Y97 ${ }^{\text {CPSF4 }}$, which is substituted with a phenylalanine residue in the worm ortholog. In addition, nine of the 10 amino acids in CPSF4 and WDR33 that form the $\pi-\pi$ stacking and hydrophobic interactions with the AAUAAA RNA element (Clerici et al. 2017) are also conserved in the CPSF4 and WDR33 worm orthologs CPSF- 4 and PFS-2 (A1:K69 ${ }^{\mathrm{CPSF} 4}$ with $\mathrm{K} 83^{\mathrm{CPSF}-4}$ and F84 ${ }^{\mathrm{CPSF} 4}$ with F98 ${ }^{\text {CPSF-4 }}$; A2: $\mathrm{H} 70^{\text {CPSF } 4}$ with $\mathrm{H} 84^{\text {CPSF-4 }}$; U3: $1156^{\text {WDR33 }}$ with $\mathrm{I} 181^{\text {PFS-2 }}$; A4: F112 ${ }^{\text {CPSF } 4}$ with F126 $6^{\text {CPSF-4 }}$ and F98 $8^{\text {CPSF } 4}$ with F112 ${ }^{\text {CPSF-4 }}$; A5: F98 ${ }^{\text {CPSF } 4}$ with F112 ${ }^{\text {CPSF-4 }}$; A6: F153 ${ }^{\text {WDR33 with }}$ F178 ${ }^{\text {PFS-2 }}$ ) (Fig. 1B; Supplemental Fig. S1). The only exception is a F43 ${ }^{\text {WDR33 }}$ substitution to a glycine residue that interacts with A6 in the worm ortholog.

CPSF3, the endonuclease that performs the cleavage reaction, has a C. elegans ortholog named CPSF-3. Both genes are conserved with an overall $57.61 \%$ identity that increases to $69.52 \%$ in the $\beta$ lactamase domain, which is the region required to perform the cleavage reaction (Fig. 1B; Supplemental Fig. S1). Specifically, all eight amino acids shown previously to form the zinc binding site required for the cleavage reaction (Mandel et al. 2006) are also conserved (D75 ${ }^{\text {CPSF3 }}$ with D74 ${ }^{\text {CPSF-3 }}$; H76 $6^{\text {CPSF3 }}$ in H75 ${ }^{\text {CPSF-3 }}$; H73 ${ }^{\text {CPSF3 }}$ in D72 ${ }^{\text {CPSF-3 }} ; \mathrm{H}_{396^{\mathrm{CPSF} 3}}$ with $\mathrm{H} 397^{\mathrm{CPSF}-3} ; \mathrm{H} 158^{\mathrm{CPSF} 3}$ with

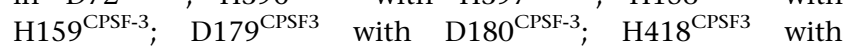
H419 ${ }^{\text {CPSF-3 }}$; E204 ${ }^{\text {CPSF3 }}$ with E205 ${ }^{\text {CPSF-3 }}$ ) (Fig. 1B; Supplemental Fig. S1). This overall similarity is also observed in most of the other members of the bona fide C. elegans CPC complex (Supplemental Fig. S1), suggesting similar structure and function.

In addition, when subjected to RNAi analysis, each of the C. elegans CPC members produced a similar strong embryonic lethal phenotype, suggesting that each of these genes may act as a complex and is required for viability (Fig. 1C; Supplemental Fig. S2).

\section{An updated 3 '-end mapping strategy}

Next, we used a genome-wide approach to improve the current version of the 3' UTRome. We refined a 3'-UTR mapping pipeline we previously developed and used in the past (Blazie et al. 2015, 2017). This approach uses raw transcriptome data as input material to identify and precisely map high-quality 3'-UTR end clusters (Fig. 2; Supplemental Fig. S3). A similar approach was previously applied to study C. elegans transcriptomes in the past (Tourasse et al. 2017).

We wanted to obtain the most accurate, saturated, and tissueindependent data set possible. To achieve this goal, we downloaded the entire collection from 2015 to 2018 of transcriptome data sets stored in the SRA (Supplemental Table S1) and processed them through our $3^{\prime}$-UTR mapping pipeline. We reasoned that this approach would lead to the identification of as many $3^{\prime}$-UTR isoforms as possible in an unbiased manner because these downloaded transcriptomes have been sequenced using both wild-type and mutant strains subjected to many different environmental conditions and covering all developmental stages with many replicates.

We downloaded a total of 1088 C. elegans transcriptome data sets ( 2 TB total raw data) (Supplemental Table S1). Most of these data sets have also been used in the past to map polyadenylation sites in C. elegans. Our 3'-UTR mapping approach extracted from these data sets approximately five million unique, high-quality poly(A) reads, which we then used for cluster preparation and mapping (Methods). We implemented very restrictive parameters for cluster identification and 3'-UTR end mapping to limit the unavoidable noise produced by using such diverse data sets as data sources (Supplemental Fig. S3). Our approach led us to map 3'UTR clusters with ultradeep coverage of several magnitudes (average cluster coverage $\sim 220 \times$ ) (Fig. 2A) and to identify 23,084 3'-UTR isoforms corresponding to 14,788 protein-coding genes. When compared to the previous 3'-UTRome v1 data set (Mangone et al. 2010), we obtained $3^{\prime}$-UTR information for an additional 3242 new protein-coding genes (4272 3'-UTR isoforms; 73\% of all protein-coding genes included in the WS250 release) (Fig. 2B,C; Supplemental Fig. S4).

\section{The C. elegans 3' UTRome v2}

Our approach produced high-quality 3'-UTR data for 14,788 C. elegans protein-coding genes (Fig. 2B). The most abundant nucleotide in C. elegans $3^{\prime}$ UTRs is a uridine, which accounts for $\sim 40 \%$ of all nucleotides in $3^{\prime}$ UTRs (Fig. 3A, top left). Adenosine nucleotides are the second most represented nucleotide class with $\sim 30 \%$ incidence (Fig. 3A, top left). Alternative polyadenylation is common but occurs at a lesser extent than what was previously published (Mangone et al. 2010; Jan et al. 2011). The majority of protein-coding genes (58\%) are transcribed with only one 3'-UTR isoform (Fig. $3 \mathrm{~A}$, bottom left) which closely resembles the previously reported $\sim 61 \%$ (Mangone et al. 2010; Jan et al. 2011). Genes with two 3'UTR isoforms are increased in occurrence when compared with past studies (32\% vs. $25 \%$ ), whereas the occurrence of genes with three or more $3^{\prime}$ UTRs is comparable with what was previously found (Fig. 3A, bottom left; Mangone et al. 2010; Jan et al. 2011).

In the case of genes with multiple $3^{\prime}$ UTRs, the canonical AAUAAA PAS site is more than two times more abundant in longer $3^{\prime}$-UTR isoforms than in shorter $3^{\prime}$-UTR isoforms, suggesting that the preparation of shorter $3^{\prime}$-UTR isoforms may be subject to regulation (Supplemental Fig. S5).

The mean $3^{\prime}$-UTR length in the 3' UTRome v2 is $215 \mathrm{nt}$ (Fig. $3 \mathrm{~A}$, top right), and the occurrence of more $3^{\prime}$-UTR isoforms per gene correlates with an overall extension in length (Fig. 3A, top right). We also note a slight correlation between 3 '-UTR length and PAS element usage, with longer $3^{\prime}$ UTRs more frequently containing variant PAS elements (Fig. 3A, bottom right). The most common PAS element in C. elegans protein-coding genes is consistently the hexamer "AAUAAA," which is present in 58.4\% of all the 3' UTRs mapped in this study (Fig. 3B, left). This element is $\sim 20 \%$ more abundant than what was previously identified in past studies (Mangone et al. 2010; Jan et al. 2011), and a slight variation of this motif is also present in genes with no canonical PAS elements (Supplemental Fig. S6). The PAS sequence is located $\sim 18 \mathrm{nt}$ from the cleavage site (Fig. 3B, right), and a buffer region of $\sim 12 \mathrm{nt}$ is present between the PAS element and the cleavage site (Fig. 3C). The cleavage site occurs almost invariably at an adenosine nucleotide, which is often preceded by a uridine nucleotide (Fig. 3C). A Gene Ontology (GO) term analysis in genes with one, two, or three $3^{\prime}$ UTR isoforms (Supplemental Fig. S7) revealed a few unique patterns with no major hits, perhaps because APA is so widespread in C. elegans and affects almost half of its known protein-coding genes.

\section{An RRYRRR motif in 3' UTRs with variant PAS elements}

We could not detect any enrichment for the UGUA motif near the cleavage site (Supplemental Fig. S8). Perhaps this element is not used in C. elegans, or the CFIm complex may recognize a variant motif not yet identified in this organism. When we aligned the 3'-ends of 3' UTRs, which contain variant PAS elements, we 

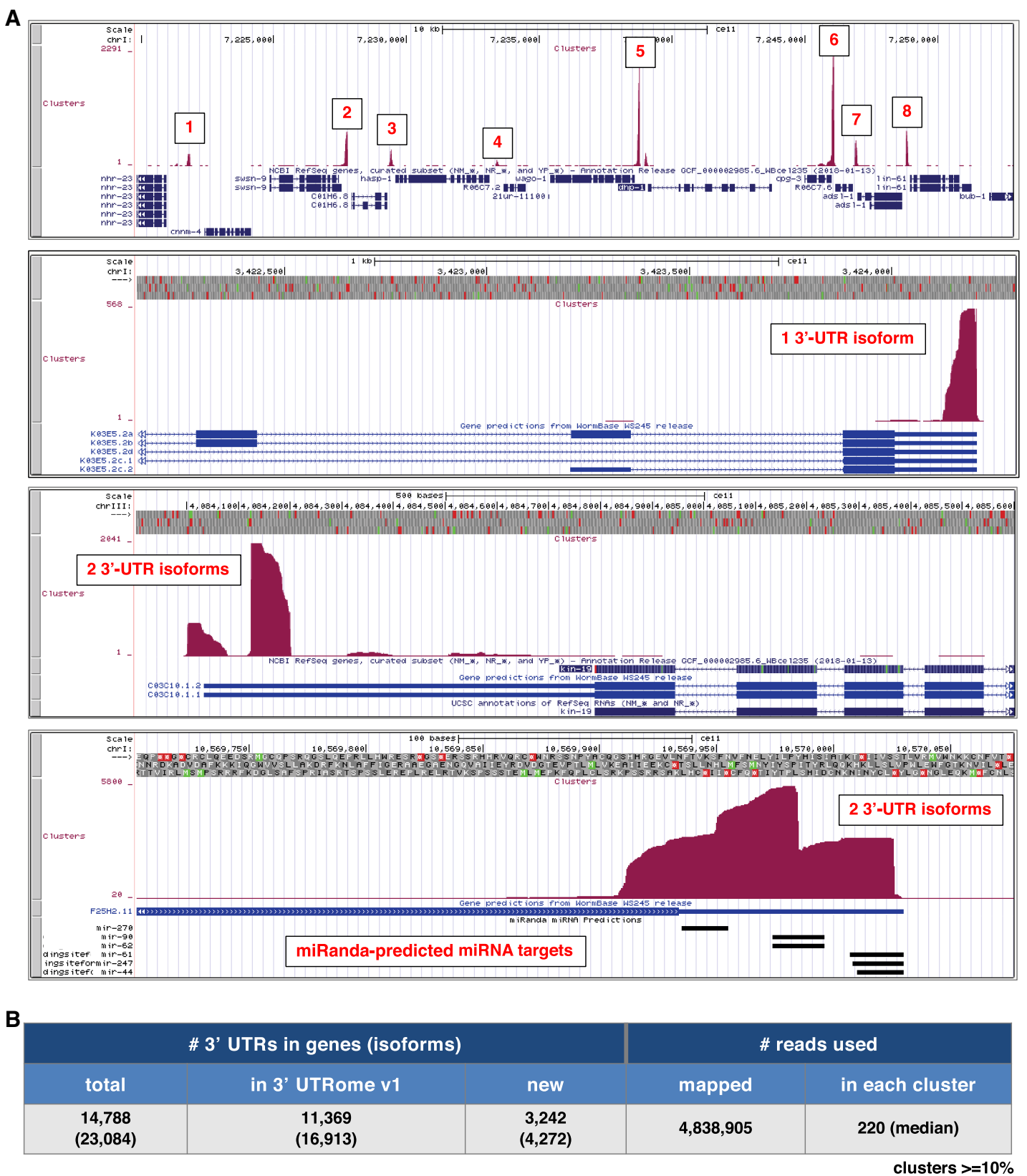

C

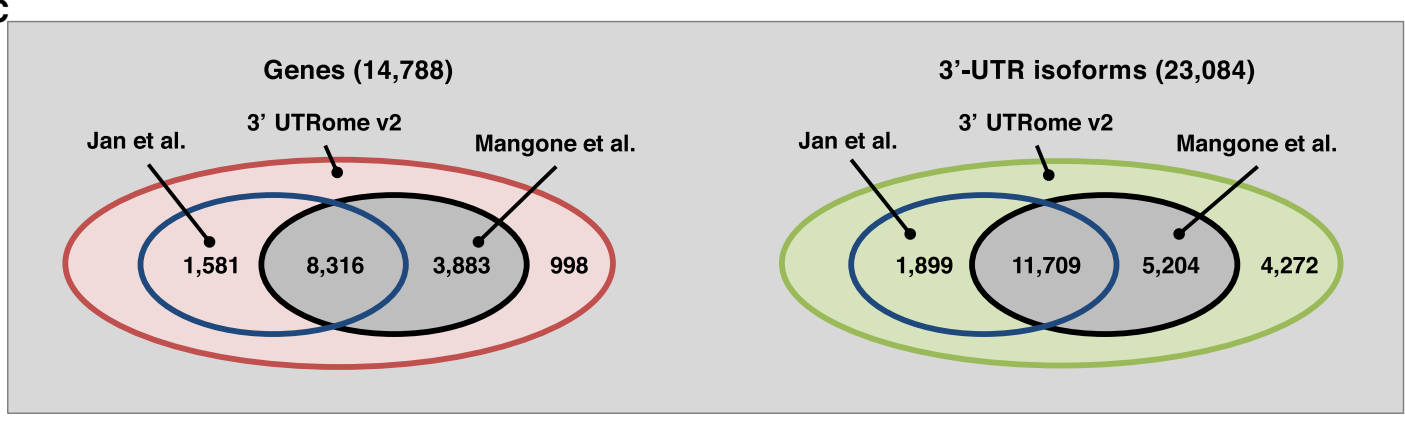

Figure 2. Cluster preparation and analysis. (A) Screenshots showing several mapped $3^{\prime}$-UTR clusters for genes with one or two 3'-UTR isoforms. MiRandapredicted miRNA targets are shown for a particular $3^{\prime}$ UTR at the bottom. (B) Summary of the $3^{\prime}$ UTRs in genes identified in this study along with the number of reads mapped and clustered for each $3^{\prime}$ UTR. (C) Comparison between the $3^{\prime}$ UTRs for genes and total isoforms mapped in this study versus the UTRome v1 (Mangone et al. 2010) and the data set from Jan et al. (2011).

\section{Genome Research}

www.genome.org 
A

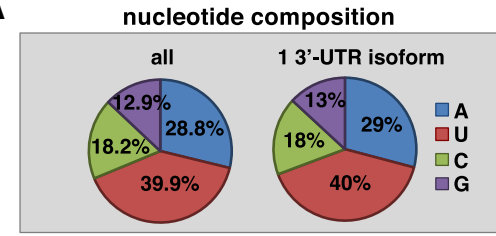

\# 3'-UTR isoforms each gene

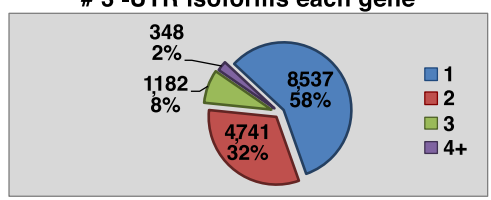

B

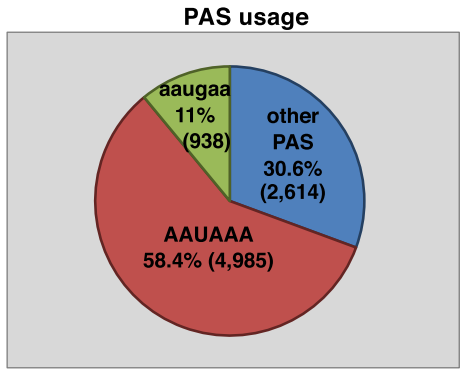

C

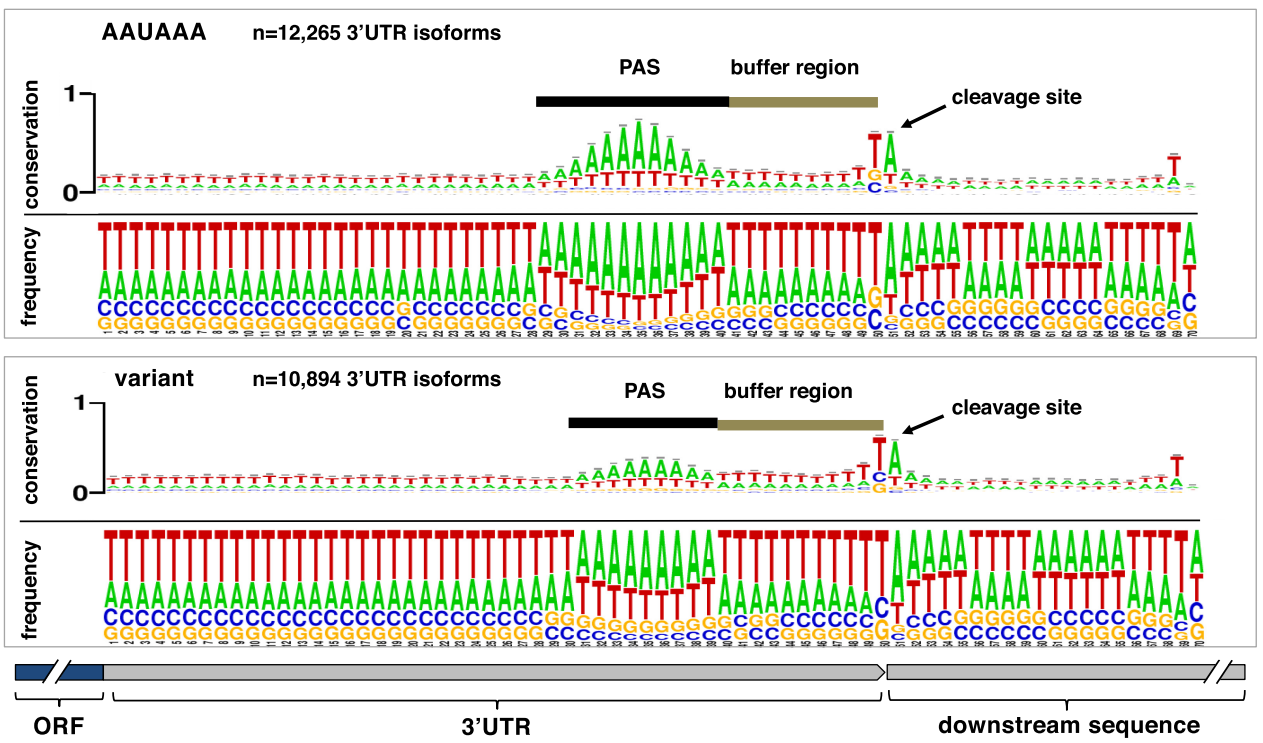

Figure 3. The worm $3^{\prime}$ UTRome v2. ( $A$, top left) Nucleotide composition of $3^{\prime}$ UTRs in the $3^{\prime}$ UTRome v2. Uridine is the most abundant nucleotide within $3^{\prime}$ UTRs for $C$. elegans. (Bottom left) The number of $3^{\prime}$-UTR isoforms in each gene, and $42 \%$ of the genes in the $3^{\prime}$ UTRome v2 possess multiple $3^{\prime}$-UTR isoforms. (Top right) $3^{\prime}$-UTR length distribution in genes expressed with one, two, or three or more $3^{\prime}$-UTR isoforms. The median $3^{\prime}$-UTR length across these data sets is $122 \mathrm{nt}$. Genes with multiple 3'-UTR isoforms are on average longer than genes with one 3'-UTR isoform. (Bottom right) Median 3'-UTR length in genes with Canonical (C) or Variant (V) PAS elements. There is a slight increase in $3^{\prime}$-UTR length in genes with variant PAS elements when compared to those with canonical PAS elements. This variation is still detected when increasing the stringency of the density of the clusters (cd) used in this analysis. $\left(B\right.$, left) PAS element usage in $3^{\prime}$ UTRs showing that $58.4 \%$ of $3^{\prime}$ UTRs use the canonical PAS element "AAUAAA," whereas the most common variant PAS element is the hexamer "AAUGAA," which occurs in 11\% of genes. (Right) The distribution of canonical PAS elements within 3' UTRs. The average distance from the PAS element to the cleavage site is $18 \mathrm{nt}$. (C) Alignment of $3^{\prime}$ UTRs at the cleavage site. This alignment in genes with both canonical and variant PAS elements reveals a region between the PAS element and the cleavage site we renamed the buffer region in which cleavage rarely occurs. The most abundant nucleotide at the cleavage site is an adenosine nucleotide preceded by a uridine nucleotide. 
noticed an enrichment of a "RRYRRR" motif which in most instances resembles a canonical AAUAAA motif with a guanine replacing the A4 nucleotide (Fig. 4A; Supplemental Fig. S6). This finding suggests that in C. elegans a "RRYRRR" element could be used when the AAUAAA hexamer is absent (Fig. 4A). We also identified other conserved elements that need to be further validated (Supplemental Fig. S9).

To better understand the molecular details of the interaction between CPSF and the PAS element, we built an atomic homology model of the worm CPSF core complex containing CPSF-1 (CPSF1), PFS-2 (WDR33), and CPSF-4 (CPSF4) (Fig. 4B; Supplemental Fig. S10). Most of this model can be superimposed to the cryo-EM structure of the human CPSF core complex (Fig. 4B; Supplemental Fig. S10).

The nucleotide-binding pocket can also be fitted into our homology model, which may implicate a conserved binding region in the C. elegans complex (Fig. 4B, right). From the structural details of the human CPSF core complex, the interactions between the RNA nucleotides and CPSF4 or WDR33 are not specific. The nucleotide binding is mainly established by the $\pi-\pi$ ring stacking force between the nucleotide bases and the residues with aromatic side chains, such as phenylalanine and tyrosine (Supplemental Fig. S10). Also, the buried area of the nucleotide-binding sites in our model was $1138 \AA$, which is similar to the nucleotide-binding pocket in the human complex (1261 $\AA$ ). The RMSD of the two models $(1.170 \AA)$ indicates a high structural similarity. As observed in the cryo-EM structure by Sun et al. (2018), no specific interactions between nucleotides and the adjacent residues were found, and the interactions between the nucleotide and adjacent residues' side chains are mostly $\pi-\pi$ ring stacking force (Supplemental Fig. S10). The actual interactions between the bound nucleotides and proteins will need to wait for the structure of the complex determined by crystallography or cryo-EM to validate it (Supplemental Fig. S10). Thus, at least in C. elegans, the selectivity of the nucleotide binding may be only at a level to the nucleotide bases, that is, pyrimidines or purines.

\section{An enrichment of adenosine nucleotide at the cleavage site}

We were intrigued by the almost invariable presence of adenosine nucleotides near the cleavage site. This enrichment becomes more evident when we sort $3^{\prime}$ UTRs with canonical PAS elements by the length of their respective buffer regions (Fig. 5A). In the case of the largest group with a buffer region of 12-13 nt, more than $20003^{\prime}$ UTRs terminate with $\sim 70 \%$ occurrence of adenosine nucleotides at the cleavage site preceded most of the time by a uridine. Because we bioinformatically removed the poly(A) sequences from the sequencing reads during our cluster preparation step, we do not have direct evidence that this last adenosine nucleotide is indeed present in the mature transcripts and used as a template for the polymerization of the poly(A) tail or that it is attached by PABPN1 during the polymerization of the poly(A) tail. The high abundance of this nucleotide at the cleavage site suggests that it is somehow important in the cleavage process.

We decided to investigate this issue further and study how precisely the raw reads produced by our cluster algorithm align to the genome. We noticed that in each gene, the cleavage rarely occurs at a unique position in the transcript. Instead, there are always slight fluctuations of the exact cleavage site, with a few percentages of reads ending a few nucleotides upstream of or downstream from the most abundant cleavage site for a given gene (Fig. 5B). Almost all the reads in each cluster terminate at an adenosine nucleotide (Fig. 5B). Also, if there are adenosine nucleotides located within shorter buffer regions, the cleavage rarely occurs at these sites. Perhaps, the large size of the CPC does not allow for the docking and the cleavage of the pre-mRNAs near the PAS element, which is optimally performed at $12-13 \mathrm{nt}$ downstream from the PAS element (Fig. 5A,B).

Next, we decided to study the role of the terminal adenosine nucleotide in the cleavage process. We reasoned that if this adenosine nucleotide indeed plays any role in the cleavage process, we should be able to alter the position of the mRNA cleavage site by mutating this residue with different purines or pyrimidines in the pre-mRNAs of selected test genes.

We selected three test genes: ges-1, Y106G6H.9, and M03A1.3. These genes are processed with a single 3 '-UTR isoform; use a single canonical PAS element; have a buffer region of 12,13 , and $14 \mathrm{nt}$, respectively; and possess a terminal uridine and adenosine nucleotide in their sequence. To capture their entire 3'-UTR region, we cloned the genomic portions of these genes spanning from their translation STOP codons to $\sim 200$ nt downstream from their cleavage sites. We then prepared several mutant $C$. elegans strains by replacing their terminal adenosine nucleotide at their cleavage site with other nucleotides. In the case of $Y 106 G 6 H .9$, we also prepared a double mutant removing an additional adenosine nucleotide downstream from the first one located at the cleavage site (Fig. 5C; Supplemental Figs. S11-S13).

We cloned these $w t$ and mutant 3 '-UTR regions downstream from a GFP reporter vector and prepared transgenic C. elegans strains that express them in the worm pharynx using the myo-2 promoter. We opted to use the pharynx promoter because it is very strong and produces a robust expression of our constructs (Supplemental Figs. S11-S13). We prepared transgenic worm strains expressing these constructs, recovered total RNAs, and tested if the absence of the terminal adenosine nucleotide in our mutants affects the position of the cleavage site using RT-PCR and a sequencing approach (Fig. 5C; Supplemental Figs. S11-S13).

We observed an overall disruption of the cleavage process, in some cases more pronounced than in others (Fig. 5C; Supplemental Figs. S11-S13). In the case of M03A1.3, the absence of the terminal adenosine nucleotide forces the cleavage complex to backtrack in $40 \%$ of the tested clones and cleave the mRNAs 3 nt upstream of the original cleavage site, but still at an adenosine nucleotide (Fig. 5C; Supplemental Fig. S11). The new cleavage site does not possess the conserved uridine upstream of the adenosine residue, suggesting that perhaps this nucleotide is not strictly required for the cleavage reaction.

In the case of $Y 106 G 6 H .9$, the single mutant does not alter the position of the cleavage site, but it activates a novel cryptic cleavage site $100 \mathrm{nt}$ upstream of the canonical cleavage site in $20 \%$ of the sequenced clones (Fig. 5C; Supplemental Fig. S12). This new site also possesses a terminal UA dinucleotide, a nonused PAS element containing the motif YRYRRR, which could still be recognized by the CPSF core complex, and a buffer region of $12 \mathrm{nt}$. In one case, the Y106G6H.9 double mutant skipped the original cleavage site but still cut at the next purine residue, which is not an adenosine in this case (Supplemental Fig. S12). In the case of ges-1, mutating the terminal adenosine does not change the cleavage pattern, although it became more imprecise (Supplemental Fig. S13).

\section{Updated miRanda predictions in C. elegans}

Next, we used our new 3' UTRome v2 data set to update miRanda miRNA target predictions. We downloaded and locally ran the

\section{Genome Research}

www.genome.org 
A

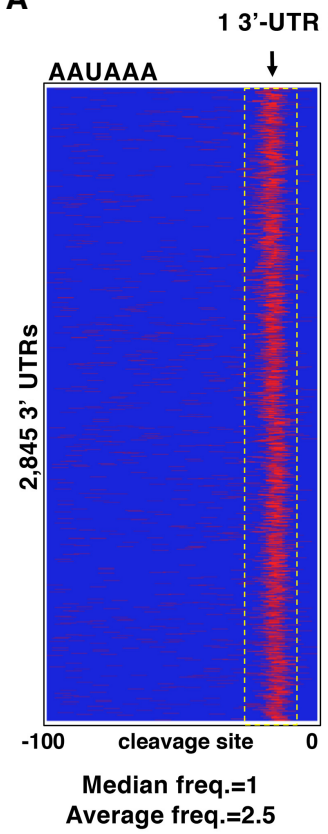

3'-UTR isoform $\square:$ RRYRRR

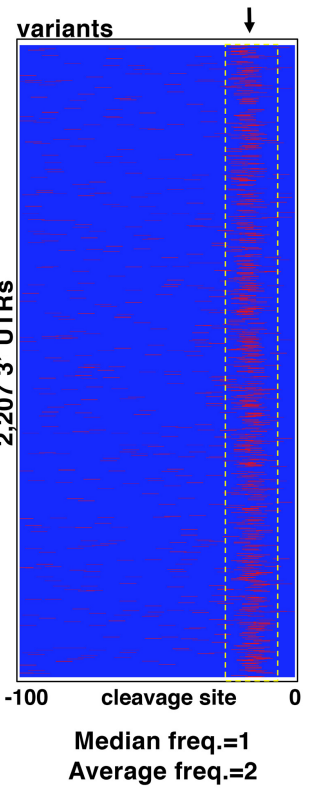

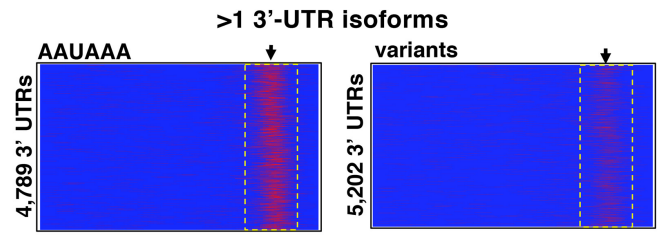

worm 3' UTRome v2

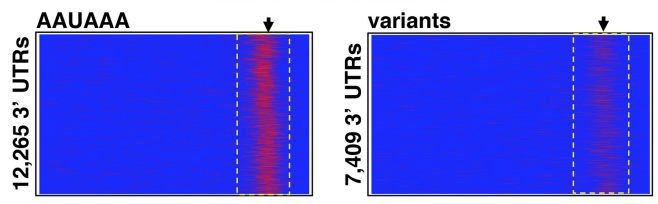

human 3' UTRome (hg38)
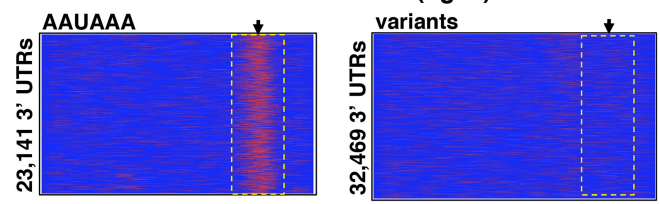

B
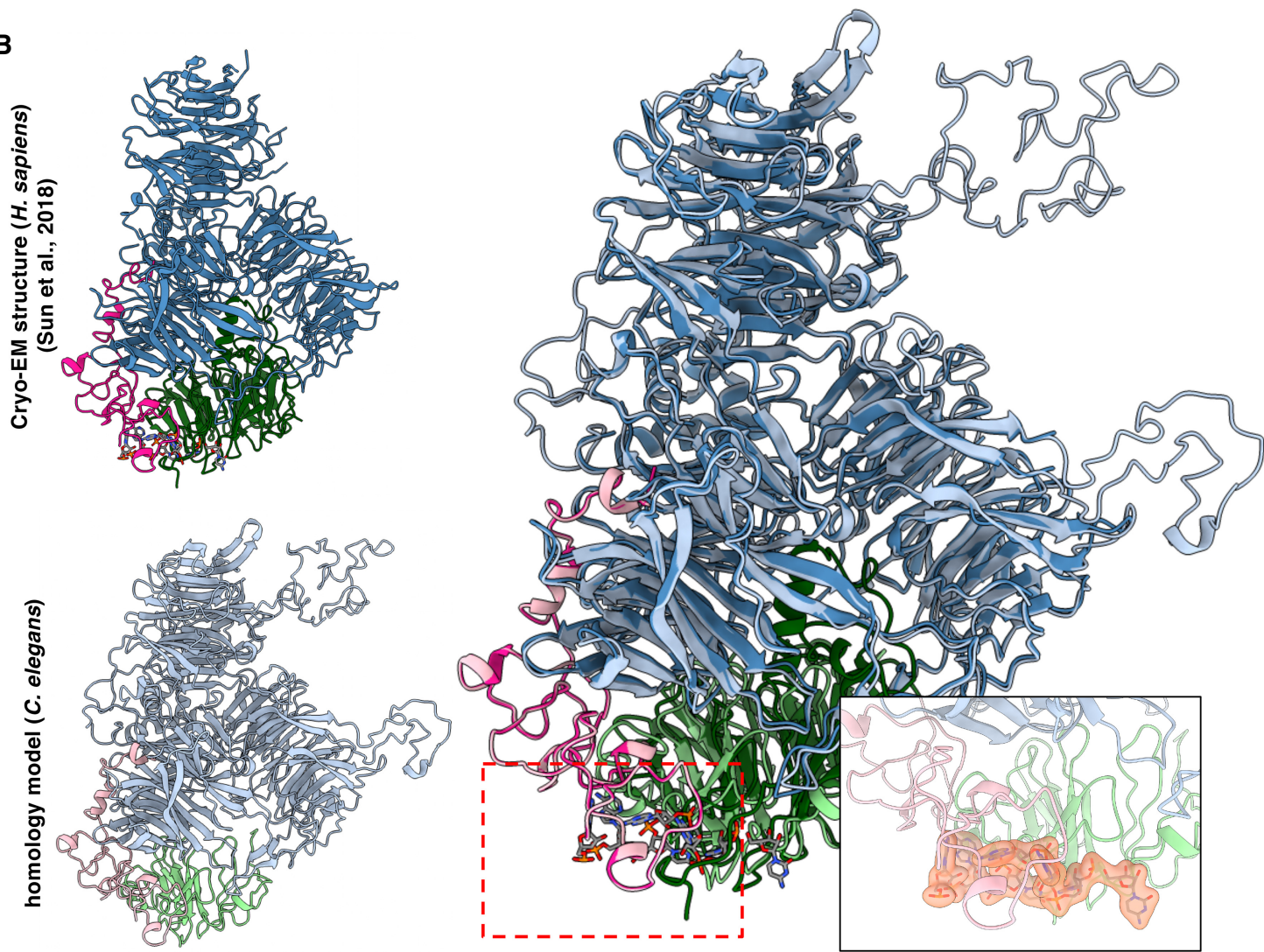

Figure 4. The sequence requirements of the C. elegans CPSF core complex. (A) PAS element usage of the RRYRRR motif. $3^{\prime}$ UTRs from the $3^{\prime}$ UTRome v2 aligned by their cleavage site in genes with canonical or variant PAS element. Instances of the motif RRYRRR are represented by the thin red bars mapped on $3^{\prime}$-UTR sequences with the coordinates -100 to $0 \mathrm{nt}$, in which $0 \mathrm{nt}$ represents the cleavage site. The spatial conservation highlighted by the yellow box of this RRYRRR motif is very strong in single 3'-UTR isoforms with canonical PAS elements and is enriched in those with variant PAS elements. This RRYRRR element is maintained in $3^{\prime}$ UTRs that have at least two isoforms, but it is not strongly represented in human 3'-UTR data (hg38) owing to the lack of their annotation. (R) Purine; (Y) pyrimidine. (B) Superimposition of the cryo-EM structure of the previously published human CPSF core complex (Clerici et al. 2018; Sun et al. 2018) to the worm CPSF core complex: CPSF-1 (CPSF1) in blue; PFS-2 (WDR33) in pink; CPSF-4 (CPSF4) in green. The PAS element binding pocket can be fitted into the homology model. The PAS element of the RNA is represented in yellow. The size and the selectivity of the nucleotide-binding pocket can fit other nucleotides as long as the motif is RRYRRR. 
A

genes w/ unique 3' UTRs with canonical PAS $=4,370$

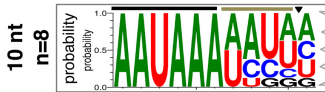

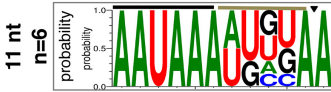

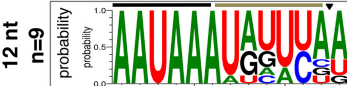

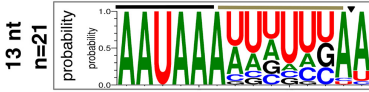

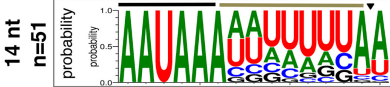

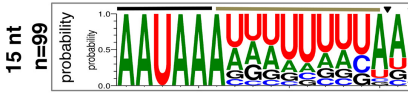

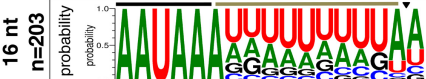

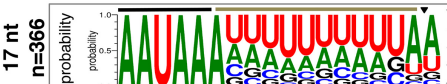

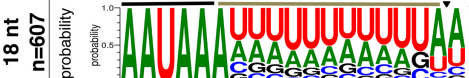

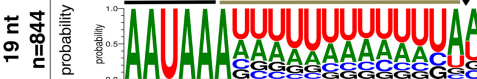

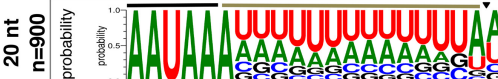

三尺高

స 'I
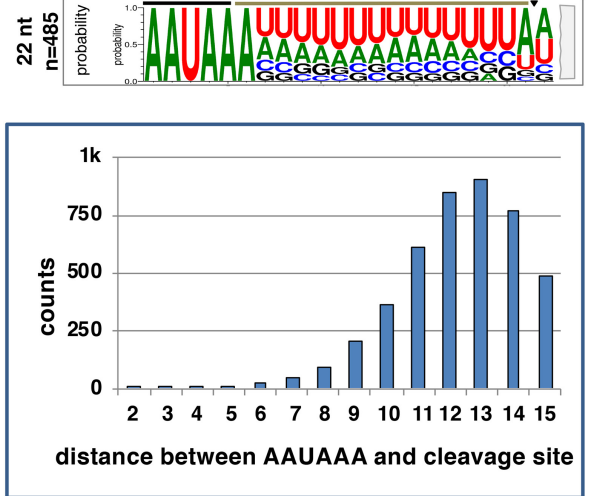

B

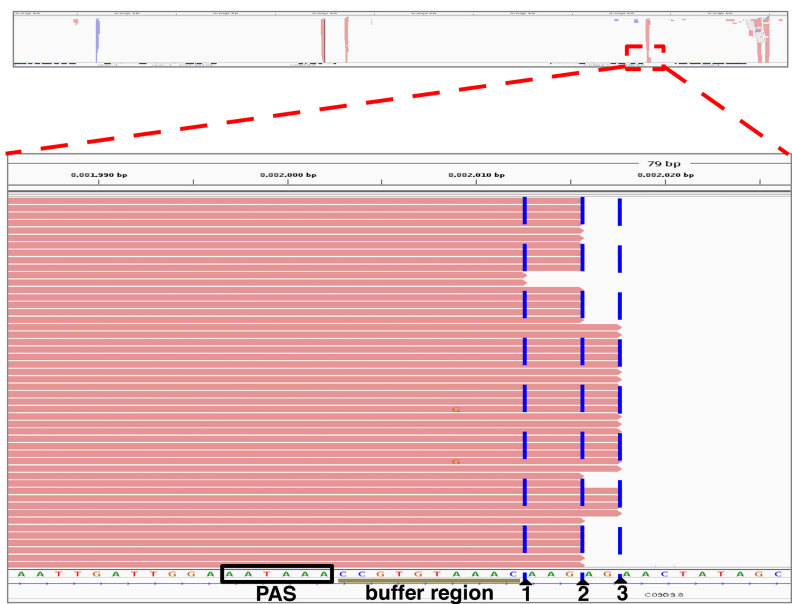

C

M03A1.3

TGAAAGGACCTGCAGTGTTTTGGGCGATTGGAGTATTCTTCTGCATTGCT GTTGCGTTGTCACTTCTTGTCGTCAATGGATATAAAAATGTATAATTATI AATGGAATTTTGGAATCTCATCTAATTTATTGATTTTATTGAATACGGGT AGTTTCTGATAATTACTTTGCATTGTAAAAAAACAAACTTTGTATGAATA AACATATTGAACATCTAAGTGCTTGCGTTTTTTTAAACTCAACTTTGGTT GCGCATATCTTGGCTCTCTTTAGTTTTTATTAAAAAATGTCAACTACAGA
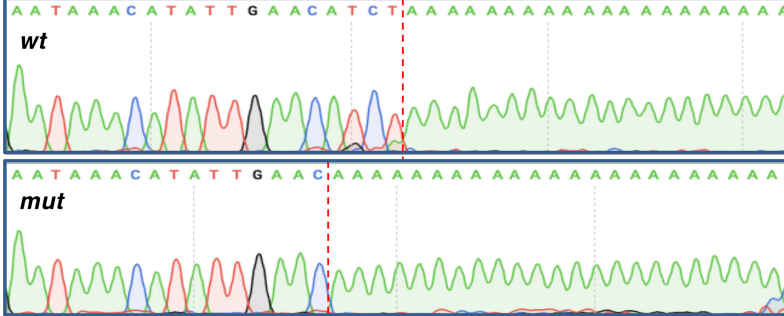

Y106G6H.9

AGAGCCACGTGCACCTTCTATAAACATCCAAAAAAACTAAATATATATTT TTTTGAAATGCAAACAACACTCCGCAGTTTTGTTTGGAAAACGAATTGGT CTACTTCTTCATAAAACATATGCGGTTCAATTGATACTTTTATTTCCATT GGAATTAAATTTAATGAATTGCTTCTTTAAATATTATTTCTATGCATCTG TTCTTCCTTTTGATTCTTCCATGAATATCTTTTTTTTATTGATCCTACAG GATCGTACAGGATCTTGTCACACTAAAGATATCTACATATTTAATAATGT TCACCTTTGTTTTCTATTCTTCATGCCAATAAAGAGAAAGTTTAATATTT TCTAGTCTGGAATTTTTATTTTTAAAAAGCTGTCAACTGACAAATTATTG TCCACGACTTCGTCTGTTATTTTTAGTGAACTAAATGTTAGATCGACAGT

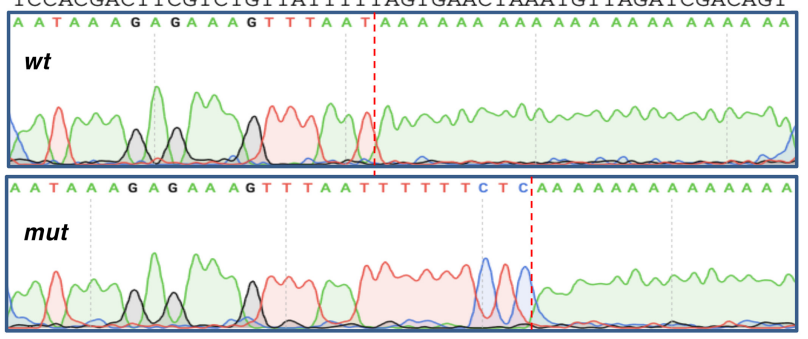

Figure 5. A terminal adenosine nucleotide is required at the cleavage site for correct cleavage. (A) Sequence logos produced from $3^{\prime}$ UTRs of genes only with 3'-UTR isoforms containing the canonical PAS element "AAUAAA" and aligned by their respective buffer region length $(n=4374)$. Two extra nucleotides are included downstream from each cut site (triangle), highlighting the terminal UA dinucleotide. The nucleotide distribution of the distance between the PAS element and the cleavage site is shown in the bar chart below. (B) Example of slight variability in the cleavage site for the gene C09G9.8. Although prevalent forms are observed, the exact cleavage site varies on several occasions, but it predominantly occurs at a different adenosine nucleotide. (C) Test of the role of the terminal adenosine nucleotide in the cleavage reaction. The $3^{\prime}$-end regions of several test genes were cloned and used to prepare transgenic C. elegans strains expressing this region with or without mutated terminal adenosine nucleotides (red, see below). The top sequence shows the test 3'-end region: (cyan) ORF; (green) translation STOP signal; (gray) 3' UTR; (red) terminal adenosine nucleotide. The PAS element is underscored. The Sanger trace files show the outcome of the cleavage site location in selected clones. Two genes are shown (M03A1.3 and Y106G6H.9). In the case of M03A1.3, the loss of the terminal adenosine nucleotide sometimes forces the CPC to backtrack and cleave the mRNAs upstream of the regular cleavage site but still at the closest adenosine nucleotide available. In the case of the gene $Y 106 \mathrm{G} 6 \mathrm{H} .9$, the loss of the terminal adenosine nucleotide forces the complex to skip the cleavage site, which sometimes occurs at the next purine nucleotide. Additional clones and more test genes are shown in Supplemental Figures S11-S13.

\section{Genome Research}

www.genome.org 
miRanda prediction software (John et al. 2004) using our new 3' UTRome v2 as a target data set. We produced two sets of predictions: one generic, which contains the entire output produced by the software; and one more restrictive, which contains only output predictions with high scores and with low E-energy scores. These two tracks have been uploaded in both the 3'-UTRome database (Mangone et al. 2008, 2010) and WormBase (Lee et al. 2018). Alternative polyadenylation was previously shown to allow genes to evade miRNA regulation (Blazie et al. 2017). To study this process in the context of miRNA targeting, we also performed a GO term analysis on the genes known to use APA that either lose or gain a miRNA target (Supplemental Fig. S14). We have uploaded this data set as Supplemental Table S4.

\section{Discussion}

Here, we used a genome-wide approach to refine and study the 3' UTRome in the nematode C. elegans. We identified 3'-UTR data for 14,788 genes corresponding to 23,084 3'-UTR isoforms, improving their annotation. We now have $3^{\prime}$-UTR data for $73 \%$ of all protein-coding genes included in the WS250 release. This data set is not complete, because we could not assign 3'-UTR data for the remaining 5554 protein-coding genes present in WS250 (Supplemental Table S4). For the majority of these genes, their 3'-UTR data were discarded by our highly stringent filters used during $3^{\prime}$-UTR cluster preparation. In addition, some of these genes may be transcribed at very low abundance and their mRNA is present below the sensitivity of our approach. Further experiments need to be performed to identify 3 '-UTR data for the remaining 5554 protein-coding genes.

Transcriptome data does not always reach the resolution needed to map 3'-ends of mRNAs at single-base resolution, because reads containing poly(A)s close to the cleavage site are generally discarded by aligners. In the case of short 3' UTRs that overlap entirely with a single sequencing read, it is possible to successfully attach a given 3 '-UTR cluster to the correct gene. However, because the majority of $3^{\prime}$ UTRs in C. elegans are longer than the average length of a single read, we do not have a continuous coverage from the translation STOP site to their $3^{\prime}$ end for most of our 3' UTRs. To attach our clusters to a given gene, we rely on a common practice that bioinformatically attaches them to the closest gene within $2000 \mathrm{nt}$ in the correct orientation (Mangone et al. 2010; Jan et al. 2011).

Alternative polyadenylation is widespread in C. elegans, with $\sim 42 \%$ of genes possessing at least two $3^{\prime}$-UTR isoforms (Fig. 3A). The PAS usage is still most commonly the hexamer "AAUAAA," which is used to process $\sim 58 \%$ of all C. elegans $3^{\prime}$ UTRs (Fig. 3B). We found that the remaining $42 \%$ possess a variation of this canonical PAS element that indeed is very similar in chemical composition and contains an "RRYRRR" motif at the same location where the PAS element is expected (Fig. 4A). We do not have direct evidence that the $\mathrm{CPC}$ recognizes this motif, but because it is so conserved, we hypothesize that in C. elegans it may provide a docking site in the absence of the canonical AAUAAA site during the cleavage reaction. Additional elements in the buffer region may play a role in this process, but this region is very rich in uridine residues (Figs. 3C, 5A), which makes the identification of the conserved signatures using common motif search software (Bailey et al. 2009) challenging. When we studied the sequence requirement of this RRYRRR motif in 3' UTRs of genes without a canonical PAS element (Supplemental Fig. S6), we found that the most conserved nucleotides are the Y3 and R6. These two nucleotides are adjacent to each other when bound to WDR33 and CPSF4 in human
(Sun et al. 2018) and form a Hoogsteen U-A base pair. This interaction is perhaps required to lock the mRNA in place by these two factors and is size dependent.

Our superimposition of the C. elegans CPSF ortholog to the human cryo-EM structure (Clerici et al. 2018; Sun et al. 2018) in Figure $4 \mathrm{~B}$ and Supplemental Figure S10, although not reinforced by experimental data, still supports our hypothesis, suggesting that in worms, the pocket used by this complex to bind the PAS element may accommodate other nucleotides as long as they have a similar chemical structure and can recapitulate the "RRYRRR" motif. In humans, the second most abundant PAS element is "AUUAAA" (Sun et al. 2018), which does not follow this guideline, suggesting that perhaps other factors can contribute to the cleavage of noncanonical PAS elements in other species.

Our analysis on the cleavage site found that the cleavage and polyadenylation machinery does not always cleave the same mRNA at the same position on the $3^{\prime}$ UTR (Fig. 5B). Although a predominant site is often chosen for each gene, a slight variation of a few nucleotides upstream of or downstream from the cleavage site is also possible. This slight variation almost invariably ends at an adenosine nucleotide in the genome, suggesting that this nucleotide is somehow "sensed" in the cleavage process.

Our mutagenesis results also support an important role for the terminal adenosine nucleotide during the cleavage reaction (Supplemental Figs. S11-S13). In those experiments, the loss of this terminal adenosine nucleotide disrupts the location of the cleavage in some cases, either activating cryptic cleavage sites or backtracking and using a different adenosine nucleotide upstream of the canonical cleavage site (Supplemental Figs. S11-S13). We did not mutate the upstream uridine residue, and we do not know its contribution, if any, to the cleavage reaction. Although we always detected its presence at the cleavage site (except in one case), more experiments need to be performed to confidently assign a role in this process.

The concept of mRNAs terminating with an adenosine nucleotide is not novel. Pioneering work using 269 vertebrate cDNA sequences has shown that $\sim 71 \%$ of these genes terminate with a CA dinucleotide element (Sheets et al. 1990). These experiments were biochemically validated a few years later using SV40 late poly(A) signal in mammalian cells in a more controlled environment (Chen et al. 1995). These experiments also showed that, at least for the case of this specific $3^{\prime}$ UTR, the cleavage could not occur closer than $11 \mathrm{nt}$ or further than $23 \mathrm{nt}$ from the PAS element (Chen et al. 1995). In this context, these findings could explain why we do not detect a terminal adenosine at the cleavage site with our double mutant Y106G6H.9, which is 27 nt downstream from the PAS element (Supplemental Fig. S12). In the case of this gene, the cleavage still occurs at a purine nucleotide, which suggests that perhaps another terminal purine can compensate for the absence of an adenosine nucleotide.

Overall, experiments in Figure 5C and Supplemental Figures S11-S13 support and expand both these initial results, showing that altering the nucleotide composition downstream from the PAS element may influence the location of the cleavage.

Our study does not have the resolution to definitely verify if this adenosine nucleotide is indeed included in the processed mRNAs or used by the CPC as a genomic mark of the cleavage site. More specifically, we do not know if this nucleotide is read by the RNA polymerase II and incorporated in the nascent mRNAs or if the machinery somehow "senses" its presence and cleaves the mRNA upstream of it. Another plausible hypothesis is that CPSF3 may cleave the mRNAs somewhere downstream 
from this terminal adenosine nucleotide, and then unknown exonucleases degrade the mRNA molecule until the first adenosine in a row is reached. Some insights may come from the process underlining histone 3 '-end formation, because CPSF3 also cleaves these poly(A)-lacking histone mRNAs. In this specific case, the enzyme is positioned near the cut site by the RNU7-1 snRNP and cuts the nascent pre-mRNA just downstream from an adenosine nucleotide (Yang et al. 2009). We speculate that perhaps CPSF3 is capable of either "sensing" this terminal adenosine nucleotide or is positioned next to it by either other members of the CPC or a not yet identified factor.

If this terminal adenosine is indeed incorporated in the premRNAs, its functional requirement is unclear. It may be used by the poly(A) polymerase enzyme as a substrate to extend the poly (A) tail after the cleavage reaction has been completed or perhaps has an unknown regulatory function. More experiments need to be performed to answer these questions.

Although we observed a terminal adenosine nucleotide in most of the mapped 3' UTRs, the cytosine nucleotide previously identified upstream of the terminal adenosine in humans is replaced with another pyrimidine nucleotide in C. elegans (uridine) (Fig. 3C), suggesting that other factors may contribute to the cleavage site decision by the CPC in higher eukaryotes.

MiRanda predictions were obsolete and needed to be updated because those present in the microrna.org database (http://www .microrna.org/microrna/home.do) were obtained using a 9-yr-old $3^{\prime}$-UTR data set. Also, before this study, WormBase (Lee et al. 2018) did not include miRNA targeting predictions in its JBrowse software.

The number of predicted miRNA targets is now decreased from 34,186 to 23,160 , mostly because several $3^{\prime}$-UTR isoforms in the 3' UTRome v1 were discarded in this new 3' UTRome v2 release. We used these new predictions to detect several instances of genes that use APA and can potentially escape miRNA targeting (Supplemental Table S3).

In conclusion, this new 3'-UTR data set, which we renamed $3^{\prime}$ UTRome v2 (Supplemental Table S5), has been uploaded to WormBase WS274 release (Lee et al. 2018) and is shown as a new track in the JBrowse tool together with updated miRanda miRNA targets. The 3' UTRome v2 expands the old 3' UTRome developed within the modENCODE Consortium, and, together with updated miRanda predictions, provides the C. elegans community with an important novel resource to investigate the RNA cleavage and polyadenylation reaction, $3^{\prime}$-UTR biology, and miRNA targeting.

\section{Methods}

\section{3'-UTR mapping pipeline}

We used the SRA toolkit from the NCBI to download raw reads from 1094 transcriptome experiments. The complete list of data sets used in this study is shown in Supplemental Table S1. We restricted the analysis to sequences produced from C. elegans transcriptomes using the Illumina platform with reads of at least 100 nt in length. At the completion of the download step, the files were unzipped and stored in our servers. We then used a custommade Perl script to extract reads containing at least 23 consecutive adenosine nucleotides at their $3^{\prime}$ end or 23 consecutive thymidine nucleotides at their $5^{\prime}$ end (Supplemental Code). This filter produced 24,973,286 mappable $3^{\prime}$-end reads. We then removed the terminal adenosine or thymidine nucleotides from these sequences, converted them to FASTQ files using the FASTX-Toolkit (http ://hannonlab.cshl.edu/fastx_toolkit/), and mapped them to the WS250 release of the C. elegans genome using Bowtie 2 algorithm with standard parameters (Langmead and Salzberg 2012). Bowtie 2 mapped 7,761,642 reads (31.08\%), which were sorted and separated based on their respective strand origin (positive or negative). We uploaded to WormBase two versions of this data set. The more stringent one, which we named "filtered," includes all these aforementioned filters and has been used in all the analyses performed in this study. A second data set, named "mild," includes $3^{\prime}$-UTR isoforms that overlap $\pm 2 \mathrm{nt}$ and have cluster reads $<5$. The complete set of $3^{\prime}$ UTRs composing the $3^{\prime}$ UTRome v2 are shown in Supplemental Table S5.

\section{Cluster preparations}

Poly(A) clusters were prepared as follows. We stored the ID, genomic coordinates, and strand orientation of each mapped read and used this information throughout the pipeline. The BAM file produced by the aligners was sorted and converted to BED format using SAMtools software ( $\mathrm{Li}$ et al. 2009). Contiguous genomic coordinates were merged using BEDTools software (Quinlan and Hall 2010) using the following command: "Bedtools merge $-\mathrm{C}$ 1 -o count - I > tmp. cluster". This new file produced the characteristic "shark fin" graph visible in Figure 2. We used several stringent filters to eliminate as much noise as possible: (1) We ignored clusters composed of less than six reads; (2) we extracted genomic DNA sequences $20 \mathrm{nt}$ downstream from the end of each cluster, but if the number of adenosine nucleotides was $>65 \%$ in the genomic sequence, we ignored the corresponding cluster and marked it as caused by mispriming during the second strand synthesis in the RT reaction; (3) we ignored clusters overlapping with other clusters in the same orientation by 2 nt or less; (4) we attached clusters to the closest gene in the same orientation, and if no gene could be identified within $2000 \mathrm{nt}$, the cluster was ignored; and (5) in cases with multiple 3'-UTR isoforms identified, we calculated the frequency of occurrence for each isoform and ignored isoforms occurring at a frequency of $<1 \%$ independently from the number of reads that form this cluster. The logo plots used to visualize our results were produced using the WebLogo 3 suite (Crooks et al. 2004).

\section{Extraction of $3^{\prime}$-UTR regions from the $C$. elegans genome}

The $3^{\prime}$ UTRs used in the experiments described in Figure $5 \mathrm{C}$ and Supplemental Figures S11-S13 were initially cloned from N2 wild-type C. elegans genomic DNA using PCR with Platinum Taq Polymerase (Invitrogen). Genomic DNA template was prepared as previously described (Blazie et al. 2017). Forward DNA primers were designed to include approximately $30 \mathrm{nt}$ upstream of the translation STOP codon and include the endogenous translation STOP codon. We used the Gateway BP Clonase II Enzyme Mix (Invitrogen) to clone the 3'-UTR region into Gateway entry vectors. The DNA primer was modified to include the attB Gateway recombination elements required for insertion into pDONR P2RP3 (Invitrogen). The reverse DNA primers were designed to end between 200 and 250 nt downstream from the RNA cleavage site and to include the reverse recombination element attB for cloning into pDONR P2RP3 (Invitrogen). At the conclusion of the recombination step, the entry vectors containing the cloned 3'-UTR regions were transformed into TOP10 competent cells (Thermo Fisher Scientific), using agar plates containing $20 \mathrm{mg} / \mu \mathrm{L}$ of kanamycin. The plasmids were then recovered, and clones were confirmed using Sanger sequencing with the M13F primer. The list of primers used in this study is available in Supplemental Table S2.

\section{Genome Research}

www.genome.org 
The comparative analysis shown in Supplemental Figure S1, the plasmid preparation and mutagenesis used in Figure 5 and Supplemental Figures S11-S13, the RNAi experiments shown in Figure 1, the preparation of the transgenic worm lines used to detect 3'-UTR cleavage skipping, the miRanda prediction, and the CPSF homology model are described in Supplemental Materials and Methods.

\section{Data access}

Strains and plasmids from this study are available upon request. All data necessary for confirming the conclusions of the paper are present within the article, figures, Supplemental Figures, and Supplemental Tables. The results of our analyses are available in WormBase (www.WormBase.org) (Lee et al. 2018) and in our 3'-UTR-centric website (www.UTRome.org).

\section{Acknowledgments}

We thank Heather Hrach for insights and review of the manuscript. We thank Gabrielle Richardson for maintaining the C. elegans strains used in this study. This work was supported by the National Institutes of Health (National Institute of General Medical Sciences) grant number 1R01GM118796.

Author contributions: H.S.S. and M.M. designed the experiments. M.M. developed and executed the bioinformatic analysis and 3'-UTR cluster preparation. H.S.S. performed the rescue experiments in Figure 5 and Supplemental Figures S11-S13. P.-L.C. performed the homology modeling in Figure 4B and Supplemental Figure S10 and helped write the manuscript. C.G. assisted with the experiments and performed the analysis in Supplemental Figure S1. S.O. contributed to the experiments in Supplemental Figures S11-S13. M.M. uploaded the results to the WormBase and UTRome.org database. M.M. and H.S.S. led the analysis and interpretation of the data, assembled the figures, and wrote the manuscript. All authors read and approved the final manuscript.

\section{References}

Bai Y, Auperin TC, Chou CY, Chang GG, Manley JL, Tong L. 2007. Crystal structure of murine CstF-77: dimeric association and implications for polyadenylation of mRNA precursors. Mol Cell 25: 863-875. doi:10 .1016/j.molcel.2007.01.034

Bailey TL, Boden M, Buske FA, Frith M, Grant CE, Clementi L, Ren J, Li WW, Noble WS. 2009. MEME SUITE: tools for motif discovery and searching. Nucleic Acids Res 37: W202-W208. doi:10.1093/nar/gkp335

Bartel DP. 2009. MicroRNAs: target recognition and regulatory functions. Cell 136: 215-233. doi:10.1016/j.cell.2009.01.002

Bartel DP. 2018. Metazoan microRNAs. Cell 173: 20-51. doi:10.1016/j.cell .2018.03.006

Blazie SM, Babb C, Wilky H, Rawls A, Park JG, Mangone M. 2015. Comparative RNA-Seq analysis reveals pervasive tissue-specific alternative polyadenylation in Caenorhabditis elegans intestine and muscles. BMC Biol 13: 4. doi:10.1186/s12915-015-0116-6

Blazie SM, Geissel HC, Wilky H, Joshi R, Newbern J, Mangone M. 2017. Alternative polyadenylation directs tissue-specific miRNA targeting in Caenorhabditis elegans somatic tissues. Genetics 206: 757-774. doi:10 .1534/genetics.116.196774

Chan SL, Huppertz I, Yao C, Weng L, Moresco JJ, Yates JR III, Ule J, Manley JL, Shi Y. 2014. CPSF4 and WDR33 directly bind to AAUAAA in mammalian mRNA 3' processing. Genes Dev 28: 2370-2380. doi:10.1101/gad .250993 .114

Chen F, MacDonald CC, Wilusz J. 1995. Cleavage site determinants in the mammalian polyadenylation signal. Nucleic Acids Res 23: 2614-2620. doi:10.1093/nar/23.14.2614

Chen F, Chisholm AD, Jin Y. 2017. Tissue-specific regulation of alternative polyadenylation represses expression of a neuronal ankyrin isoform in C. elegans epidermal development. Development 144: 698-707. doi:10 $.1242 /$ dev. 146001
Clerici M, Faini M, Aebersold R, Jinek M. 2017. Structural insights into the assembly and polyA signal recognition mechanism of the human CPSF complex. eLife 6: e33111. doi:10.7554/eLife.33111

Clerici M, Faini M, Muckenfuss LM, Aebersold R, Jinek M. 2018. Structural basis of AAUAAA polyadenylation signal recognition by the human CPSF complex. Nat Struct Mol Biol 25: 135-138. doi:10.1038/s41594017-0020-6

Crooks GE, Hon G, Chandonia JM, Brenner SE. 2004. WebLogo: a sequence logo generator. Genome Res 14: 1188-1190. doi:10.1101/gr.849004

Diag A, Schilling M, Klironomos F, Ayoub S, Rajewsky N. 2018. Spatiotemporal m(i)RNA architecture and $3^{\prime}$ UTR regulation in the $C$. elegans germline. Dev Cell 47: 785-800.e8. doi:10.1016/j.devcel.2018 .10 .005

Gerstein MB, Lu ZJ, Van Nostrand EL, Cheng C, Arshinoff BI, Liu T, Yip KY, Robilotto R, Rechtsteiner A, Ikegami K, et al. 2010. Integrative analysis of the Caenorhabditis elegans genome by the modENCODE project. Science 330: $1775-1787$. doi:10.1126/science.1196914

Haenni S, Ji Z, Hoque M, Rust N, Sharpe H, Eberhard R, Browne C, Hengartner MO, Mellor J, Tian B, et al. 2012. Analysis of C. elegans intestinal gene expression and polyadenylation by fluorescence-activated nuclei sorting and 3 '-end-seq. Nucleic Acids Res 40: 6304-6318. doi:10 .1093/nar/gks282

Hwang HW, Park CY, Goodarzi H, Fak JJ, Mele A, Moore MJ, Saito Y, Darnell RB. 2016. PAPERCLIP identifies microRNA targets and a role of CstF64/ 64tau in promoting non-canonical poly(A) site usage. Cell Rep 15: 423435. doi:10.1016/j.celrep.2016.03.023

Jan CH, Friedman RC, Ruby JG, Bartel DP. 2011. Formation, regulation and evolution of Caenorhabditis elegans 3'UTRs. Nature 469: 97-101. doi:10 .1038 /nature09616

John B, Enright AJ, Aravin A, Tuschl T, Sander C, Marks DS. 2004. Human microRNA targets. PLoS Biol 2: e363. doi:10.1371/journal.pbio.0020363

Kaufmann I, Martin G, Friedlein A, Langen H, Keller W. 2004. Human Fip1 is a subunit of CPSF that binds to U-rich RNA elements and stimulates poly(A) polymerase. EMBO $J$ 23: 616-626. doi:10.1038/sj.emboj .7600070

Kühn U, Wahle E. 2004. Structure and function of poly(A) binding proteins. Biochim Biophys Acta 1678: 67-84. doi:10.1016/j.bbaexp.2004.03.008

Langmead B, Salzberg SL. 2012. Fast gapped-read alignment with Bowtie 2. Nat Methods 9: 357-359. doi:10.1038/nmeth.1923

Lee RYN, Howe KL, Harris TW, Arnaboldi V, Cain S, Chan J, Chen WJ, Davis P, Gao S, Grove C, et al. 2018. WormBase 2017: molting into a new stage. Nucleic Acids Res 46: D869-D874. doi:10.1093/nar/gkx998

Li H, Handsaker B, Wysoker A, Fennell T, Ruan J, Homer N, Marth G, Abecasis G, Durbin R; 1000 Genome Project Data Processing Subgroup. 2009. The Sequence Alignment/Map format and SAMtools. Bioinformatics 25: 2078-2079. doi:10.1093/bioinformatics/btp352

Mandel CR, Kaneko S, Zhang H, Gebauer D, Vethantham V, Manley JL, Tong L. 2006. Polyadenylation factor CPSF-73 is the pre-mRNA $3^{\prime}$ end-processing endonuclease. Nature 444: 953-956. doi:10.1038/ nature 05363

Mangone M, Macmenamin P, Zegar C, Piano F, Gunsalus KC. 2008 UTRome.org: a platform for 3'UTR biology in C. elegans. Nucleic Acids Res 36: D57-D62. doi:10.1093/nar/gkm946

Mangone M, Manoharan AP, Thierry-Mieg D, Thierry-Mieg J, Han T, Mackowiak SD, Mis E, Zegar C, Gutwein MR, Khivansara V, et al. 2010. The landscape of C. elegans 3'UTRs. Science 329: 432-435. doi:10.1126/science.1191244

Martin G, Gruber AR, Keller W, Zavolan M. 2012. Genome-wide analysis of pre-mRNA $3^{\prime}$ end processing reveals a decisive role of human cleavage factor I in the regulation of $3^{\prime}$ UTR length. Cell Rep 1: 753-763. doi:10 .1016/j.celrep.2012.05.003

Matlin AJ, Clark F, Smith CW. 2005. Understanding alternative splicing: towards a cellular code. Nat Rev Mol Cell Biol 6: 386-398. doi:10.1038/ nrm1645

Mayr C, Bartel DP. 2009. Widespread shortening of $3^{\prime}$ UTRs by alternative cleavage and polyadenylation activates oncogenes in cancer cells. Cell 138: 673-684. doi:10.1016/j.cell.2009.06.016

Pérez Cañadillas JM, Varani G. 2003. Recognition of GU-rich polyadenylation regulatory elements by human CstF-64 protein. EMBO J 22: 2821 2830. doi:10.1093/emboj/cdg259

Quinlan AR, Hall IM. 2010. BEDTools: a flexible suite of utilities for comparing genomic features. Bioinformatics 26: 841-842. doi:10.1093/bioinfor matics/btq033

Ryan K, Calvo O, Manley JL. 2004. Evidence that polyadenylation factor CPSF-73 is the mRNA $3^{\prime}$ processing endonuclease. RNA 10: $565-573$. doi:10.1261/rna.5214404

Sheets MD, Ogg SC, Wickens MP. 1990. Point mutations in AAUAAA and the poly (A) addition site: effects on the accuracy and efficiency of cleavage and polyadenylation in vitro. Nucleic Acids Res 18: 5799-5805. doi:10 $.1093 / \mathrm{nar} / 18.19 .5799$ 
Stein L, Sternberg P, Durbin R, Thierry-Mieg J, Spieth J, 2001. WormBase: network access to the genome and biology of Caenorhabditis elegans. Nucleic Acids Res 29: 82-86. doi:10.1093/nar/29.1.82

Sun Y, Zhang Y, Hamilton K, Manley JL, Shi Y, Walz T, Tong L. 2018. Molecular basis for the recognition of the human AAUAAA polyadenylation signal. Proc Natl Acad Sci 115: E1419-E1428. doi:10.1073/pnas .1718723115

Takagaki Y, Manley JL. 2000. Complex protein interactions within the human polyadenylation machinery identify a novel component. Mol Cell Biol 20: 1515-1525. doi:10.1128/MCB.20.5.1515-1525.2000

Tian B, Graber JH. 2012. Signals for pre-mRNA cleavage and polyadenylation. Wiley Interdiscip Rev RNA 3: 385-396. doi:10.1002/wrna .116

Tian B, Manley JL. 2017. Alternative polyadenylation of mRNA precursors. Nat Rev Mol Cell Biol 18: 18-30. doi:10.1038/nrm.2016.116

Tourasse NJ, Millet JRM, Dupuy D. 2017. Quantitative RNA-seq meta-analysis of alternative exon usage in C. elegans. Genome Res 27: 2120-2128. doi:10.1101/gr.224626.117

West SM, Mecenas D, Gutwein M, Aristizábal-Corrales D, Piano F, Gunsalus KC. 2018. Developmental dynamics of gene expression and alternative polyadenylation in the Caenorhabditis elegans germline. Genome Biol 19: 8. doi:10.1186/s13059-017-1369-x
Yang XC, Sullivan KD, Marzluff WF, Dominski Z 2009. Studies of the $5^{\prime}$ exonuclease and endonuclease activities of CPSF-73 in histone pre-mRNA processing. Mol Cell Biol 29: 31-42. doi:10.1128/MCB.00776-08

Yang Q, Gilmartin GM, Doublie S. 2010. Structural basis of UGUA recognition by the Nudix protein $\mathrm{CFI}_{\mathrm{m}} 25$ and implications for a regulatory role in mRNA 3' processing. Proc Natl Acad Sci 107: 10062-10067. doi:10 $.1073 /$ pnas.1000848107

Yang Q, Coseno M, Gilmartin GM, Doublié S. 2011. Crystal structure of a human cleavage factor $\mathrm{CFI}_{\mathrm{m}} 25 / \mathrm{CFI}_{\mathrm{m}} 68 / \mathrm{RNA}$ complex provides an insight into poly(A) site recognition and RNA looping. Structure 19: 368-377. doi:10.1016/j.str.2010.12.021

Yang W, Hsu PL, Yang F, Song JE, Varani G. 2018. Reconstitution of the CstF complex unveils a regulatory role for CstF-50 in recognition of 3'-end processing signals. Nucleic Acids Res 46: 493-503. doi:10.1093/nar/ gkx1177

Zhu Y, Wang X, Forouzmand E, Jeong J, Qiao F, Sowd GA, Engelman AN, Xie X, Hertel KJ, Shi Y. 2018. Molecular mechanisms for CFIm-mediated regulation of mRNA alternative polyadenylation. Mol Cell 69: 62-74.e4. doi:10.1016/j.molcel.2017.11.031

Received July 17, 2019; accepted in revised form October 10, 2019.

\section{Genome Research}

www.genome.org 


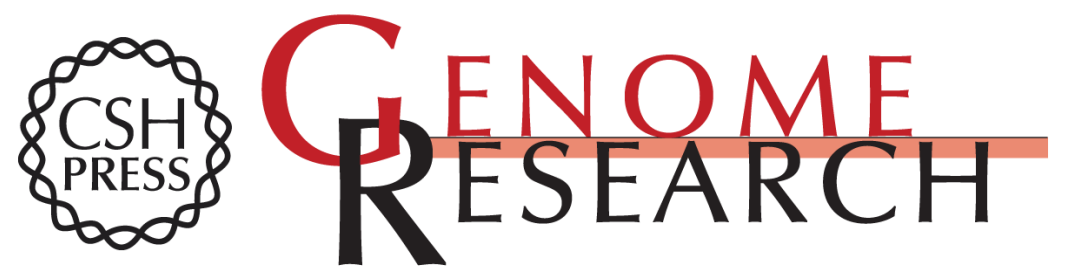

\section{The C. elegans 3' UTRome v2 resource for studying mRNA cleavage and polyadenylation, 3 '-UTR biology, and miRNA targeting}

Hannah S. Steber, Christina Gallante, Shannon O'Brien, et al.

Genome Res. 2019 29: 2104-2116 originally published online November 19, 2019

Access the most recent version at doi:10.1101/gr.254839.119

Supplemental
Material http://genome.cshlp.org/content/suppl/2019/11/19/gr.254839.119.DC1

References This article cites 45 articles, 13 of which can be accessed free at:

http://genome.cshlp.org/content/29/12/2104.full.html\#ref-list-1

Open Access Freely available online through the Genome Research Open Access option.

Creative This article, published in Genome Research, is available under a Creative

Commons Commons License (Attribution-NonCommercial 4.0 International), as described at

License http://creativecommons.org/licenses/by-nc/4.0/.

Email Alerting Receive free email alerts when new articles cite this article - sign up in the box at the Service top right corner of the article or click here.

\section{Affordable, Accurate Sequencing.}

To subscribe to Genome Research go to:

https://genome.cshlp.org/subscriptions

(C) 2019 Steber et al.; Published by Cold Spring Harbor Laboratory Press 